\title{
Learning About Letters through Handwriting Practice
}

Deborah Y. Zemlock, Department of Psychological and Brain Sciences, Indiana University - Bloomington

Faculty Mentor: Dr. Karin James

\section{ABSTRACT}

The influence of visual-motor experiences with written symbols on pre-reading abilities, such as letter knowledge, have been shown to be facilitatory in both correlational studies on very young children and in experimental studies on older children. However, it is not known whether any fine-motor practice will create this benefit, whether it is specific to writing letters, or whether certain ages would benefit most from handwriting practice. Here, we hypothesized that immature fine-motor skill that produces variable forms may be crucial to the beneficial effects of handwriting training - predicting the younger children would benefit more from the training than older children. Preschool-aged children, ages three to five years, were divided into two experimental groups (letter-writing, digit-writing) in a 2x2x2 design: TIME (pre, post), AGE (younger, older), and CONDITION (letter-writing, digit-writing). Each group received six weeks of training. The letter-writing and digit-writing groups practiced writing letters (A-Z) or single digits (0-9), respectively, four times per week. Before and after the training period, each group received assessments targeting letter knowledge directly. We predicted that the younger age group, compared to the older age group, in the letter-writing condition would score significantly higher on the letter knowledge tasks at post-test than at pre-test and that this effect would not occur in the digit-writing group. Results demonstrated that the younger children did show a significantly greater improvement in letter recognition skills than the older age group, but this effect held for both the letter-and digit- writing groups. These results suggest, therefore, that any fine-motor practice at a young age can facilitate letter knowledge.

KEYWORDS: handwriting, variable exemplars, letters, symbols, perception, motor development

\section{BACKGROUND}

T The ability to quickly and accurately recognize individual letters lies at the base of fluent reading ability, and fluent reading ability is foundational to academic success. However, 65 percent of U.S. children remain unable to read at a proficient level by the fourth grade, 16 percent of whom will fail to graduate high school (Hernandez, 2012; National Assessment of Educational Progress, 2013). Of all prereading skills, letter recognition is the strongest predictor of successful reading acquisition in fourth grade and is facilitated by experience hand-printing individual letters (James, 2010; James \& Engelhardt, 2012; Longcamp, Zerbato-Poudou, \& Velay, 2005; Scanlon \& Vellutino, 1996; Snow, Burns, \& Griffin, 1998). Surprisingly, preliterate children in typical U.S. schools spend less than twenty minutes each day on activities targeting pre-reading skills, only one minute of which is spent on handwriting activities (Pelatti, Piasta, Justice \& O'Connell, 2014). Increasing the prevalence of handwriting practice in preschool curriculums may lead to earlier attainment of pre-reading skills and, in turn, facilitate successful reading acquisition.

Although we have some evidence that early handwriting facilitates pre-literacy skill, we don't know why this may occur. There are several possibilities. We have proposed two possible alternatives. The first is the possibility that the visual output of letter production when motor skills are poor results in variable instances of a named letter - the child sees their own 'messy' productions and categorizes them into a forming category (Li \& James, in press). Another possibility is that the actual fine-motor skill of producing letters may serve to augment a visual representation of letters and, by virtue of efferent copies, may facilitate letter knowledge (James, 2010; James \& Engelhardt, 2012; Longcamp et al., 2005).
The first idea that the variable perceptual output facilitates category learning and letter knowledge is supported by a study that showed children retain significantly more object names for a given category of objects (e.g., cups) when they have experience with a variety of exemplars from that category. This indicates that experiencing within category variability (variability in exemplars) is important for children to recognize particular instances of that object category (e.g., recognizing a coffee cup and a teacup as cups; Horst, Twomey \& Ranson, 2013). As experience with the category increases, within-category differences become more distinct (Goldstone, 1994). So when children write letters in a different way each time, they are pairing a variety of exemplars to the presented stereotypical letter, and this pairing should increase their ability to recognize instances of that letter category over time.

Recognizing letters is a perceptual task that requires an ability to discriminate between perceptually similar letter categories (e.g., T vs. P). Writing and viewing a variety of symbols within a category has been shown to increase recognition. Visually studying examples of a target symbol category in variable fonts (e.g., $\Psi$ ) has been shown to improve recognition more than visually studying a target symbol category in only one stereotypical font (Li \& James, in press). In this study, across six conditions, only the participants in conditions that practice variable fonts showed improvement, regardless of whether they practiced motor productions or visual perception. This suggests that the variable output produced during early handwriting may be the key to the facilitatory effects that this manual skill has on letter knowledge.

The second idea that any task that involves visual and motor systems will facilitate learning is supported by research suggesting that manual manipulation may be a particularly 
effective pathway to object knowledge (James \& Gauthier, 2006; Longcamp et al., 2005; Longcamp, Anton, Roth \& Velay, 2003; Molfese, Beswick, Molnar \& Jacobi-Vessels, 2006). Symbols (e.g., letters or digits) are a unique object type that are very rarely manipulated manually, except in the specific case of handwriting when symbols are formed feature-byfeature. Studies have also shown that letter perception tasks activate brain regions that are also known to be activated in letter-writing tasks, suggesting that practicing with visuomotor integration, such as handwriting, can later activate both visual and motor systems when only visual stimuli is present. This is potentially because the information was encoded and retrieved through two modes (James \& Gauthier, 2006; Longcamp et al., 2003). Furthermore, Molfese, Beswick, Molnar, \& Jacobi-Vessels (2006) found a correlation between quality of produced letters during early printing and letter naming scores in preschool children who were just learning to understand letters.

In an experimental study, Longcamp, Zerbato-Poudou, \& Velay (2005) investigated how motor practice affected letter learning and found that handwriting practice led to a greater increase in letter recognition ability over typing practice. Children were divided into three experimental groups: letterwriting, letter-typing and letter-visual. Note that typing letters restricts the visual experience of the letter category to the single exemplar on the keypad, whereas printing offers children the perception of variable category exemplars (i.e., messy letters). Children were also divided into three age groups from three to five years: older, middle, and younger. After three weeks of training, the older letter-writing group improved the most in letter recognition compared to the typing or visual training groups. The authors suggested that the younger children did not have enough motor development to copy the letters accurately enough to benefit recognition.

This interpretation presents a conundrum: if variability is good for category learning and if the younger children produced more variable instances of letters due to their immature motor systems, then why did only the older children benefit from the training? One hypothesis is that the younger age group required more practice during training: perhaps the benefit of learning variable exemplars of a category requires that the learner view a wide variety of exemplars during learning. An alternate explanation is that the younger children's productions were simply too variable for them to extract commonalities among their productions. The category learning literature supports this interpretation as too much variability is not helpful if the learner cannot extract common features (Horst et al., 2013; Goldston, Medin \& Gentner, 1991; Twomey \& Horst, 2011; Twomey, Ranson \& Horst, 2013).

Therefore, although letter writing facilitates letter knowledge in older children, we do not know whether this effect is due to younger children producing too much variability in their letter productions due to their immature motor control or whether the younger age group simply requires additional training. This study sought to address this issue by increasing the training time in younger and older children to determine whether the additional training would facilitate letter recognition in the younger children.

We tested the two ideas presented above by having younger and older children learn letters and digits through handwriting practice. We address the idea that variable output may be crucial for younger children by testing both younger (messier productions) and older (neater productions) children. We also test the idea that any fine-motor skill coupled with visual perception during learning will facilitate the learning by comparing letter recognition ability in two motor training groups. This is an indirect measure of the hypothesis that stated perhaps increased fine-motor skill itself would facilitate recognition ability (Longcamp et al., 2005), and this could be achieved by digit writing as well as letter writing.

\section{METHODS \\ Participants}

Thirty-two children, 18 girls and 14 boys, with a mean age of 4.57 years $(S D=0.70)$ and an age range from 3.14 to 5.75 years, practiced letter-writing and digit-writing weekly for a total of six weeks. Two schools participated in this study; both are private preschools located in Bloomington, IN in which six children came from one school and 26 from the other. Both schools draw from similar, middle-to highincome households. All testing and training were conducted in the participants' schools after receiving informed consent from parents in cooperation with school administrators. All procedures were approved by the IRB at Indiana University.

\section{Procedure}

The procedure consisted of a pre-test, a training period, and a post-test. The pre-test was administered during the first week. The training period lasted from week two through week seven. During the training period, participants practiced writing 26 symbols, either letters or digits, four times per week. The post-test was given during the eighth week.

Pre-test. The pre-test consisted of three categories letter naming, letter recognition, and letter categorization - to be used together as a composite baseline score of letter knowledge and a suite of standardized assessments to be used as descriptive measures of the motor ability and pre-literacy level in our sample. The pre-test assessments were given in two sessions: the Symbol Knowledge Testing Session and the Standardized Testing Session, each lasting 25 to 45 minutes.

Standardized testing session. Visual perception, motor coordination, visual-motor integration, phonological processing skills, and pre-literacy levels were assessed using nine standardized assessments. Three Beery-Buktenica developmental tests to assess visual-motor integration, visual perception, and motor coordination; the La Rue Reading Skills Assessment to assess letter and digit knowledge; Rhyme Awareness and Sound Awareness sub-tests of the PiPA to assess phonological skills; and the Visual Discrimination sub-test of the Bader Reading \& Language Inventory to assess letter and word recognition (Bader, 1983; Beery, Buktenica, \& Beery, 2010; Dodd, 2008; LaRue, 2014). The tests in this 
session were not analyzed in the current study but will be used to determine the distribution of the data at the pre-test.

Letter Knowledge testing session. Four additional assessments measured letter and knowledge: letter naming, letter copying, letter recognition, and letter categorization tasks.

Letter Naming task. Participants named 26 letters in Zaner-Bloser typed font from $2.25 \times 2.75$ inch cards presented in a pseudo-randomized order.

Letter Recognition task. Participants performed a fouralternative forced-choice (4-AFC) task in which they were required to select a stereotypical typed letter exemplar from three other choices when presented with a variable handwritten letter exemplar. This method has been used in previous studies (James, 2010; James \& Engelhardt, 2012; Longcamp, Zerbato-Poudou, \& Velay, 2005). Twenty-six handwritten letters from 4.25 x 5.5 inch index cards were presented one at a time. Children were required to match the letter to one of four presented options: (1) the correct choice, which was a typed letter in Zaner-Bloser font; (2) the false choice, which was a typed pseudo-letter created by rearranging the features of the target letter; (3) another typed letter similar in shape to the correct choice; and (4) a typed mirror reversal of the correct choice. For the 11 letters that cannot be reversed (e.g., M), a "matched" false choice option that was created using the same method as (2) was presented;

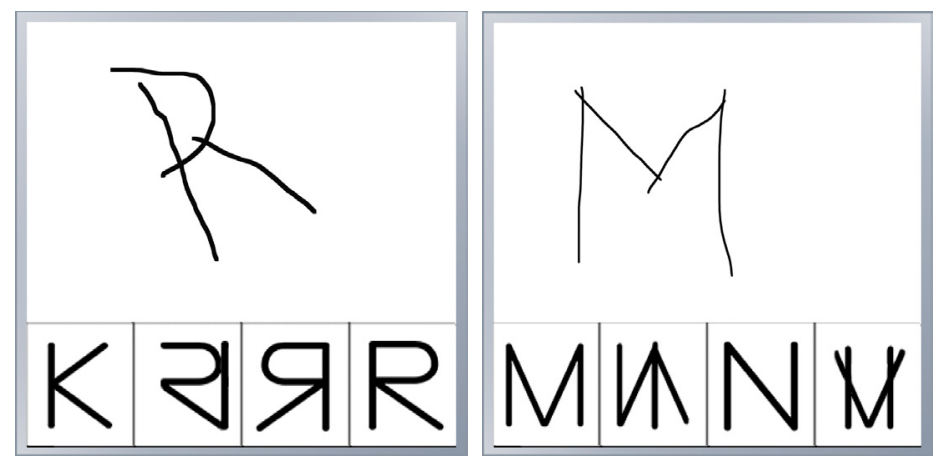

Figure 1. Sample Letter Recognition task trials. On the left is an example of a trial in the Letter Recognition task in which a reversal is included. On the right is an example of a trial in the Letter Recognition task in which a letter cannot be reversed and a "matched" false choice option is presented instead (the fourth choice).

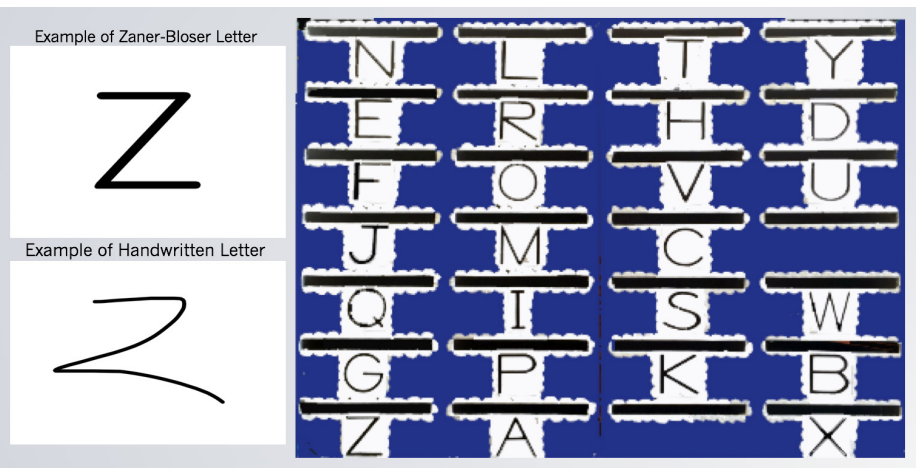

Figure 2. Depiction of the Letter Categorization task. This figure illustrates the Letter Categorization task and the two types of sorting cards: typed and handwritten. however, the results more closely resembled the correct choice, just as the reversal would bear a closer resemblance to the correct choice than a false choice (see Figure 1).

Letter Categorization tasks. Participants sorted $264.25 \mathrm{x}$ 5.5 inch cards, 17 with handwritten letter examples and nine with Zaner-Bloser typed letters, into a "mailbox" with 28 slots, of which 26 were labeled with letter cards in Zaner-Bloser font measuring $2.25 \times 2.75$ inches (see Figure 2). The extra two slots were left blank. Participants were allowed to "deliver" to those slots if they didn't know where else to place them.

The handwritten samples used in both the Letter Recognition and Letter Categorization tasks were taken from an ongoing study collecting handwriting samples from subjects ages three to five years. The quality of the handwritten sample was determined by consensus of two experimenters with an agreement of 96 percent. No subject was given the same set of handwritten samples twice and the ordering of the samples within the sets was counterbalanced between subjects.

Training. The training period began on week two and ended at week seven, lasting for a total of six weeks. To decrease experimenter time, participants completed training sessions in groups of two or three. Participants were randomly assigned to one of two experimental groups and never practiced with participants from the other experimental group.

Experimental groups. Participants were randomly assigned to one of two groups: letter-writing and digit-writing. Both training conditions wrote either single letters (A-Z) or single digits (0-9) four times each week in workbooks. Digits were repeated so that each group practiced with 26 symbols each week.

Letter-writing. Twenty-six letters were randomly divided into two sets of 13 each. Those sets were then each ordered three different ways to make three different orders for the first 13 letters $(1.1,1.2,1.3)$ and three different orders for the last 13 letters $(2.1,2.2,2.3)$ (see Figure 3).

Digit-writing. Each of the worksheet sets had 13 digits each. Those sets were then each ordered three different ways to make three different orders for the first 13 letters $(1.1,1.2$, 1.3) and three different orders for the last 13 letters $(2.1,2.2$, 2.3). All 10 digits appear once in every worksheet, and three digits were repeated (see Figure 3).

Workbooks. Symbols were presented in Zaner-Bloser typed

\begin{tabular}{|c|c|c|c|c|c|}
\hline Set & Set & Set & Set & Set & Set \\
$\mathbf{1 . 1}$ & $\mathbf{1 . 2}$ & $\mathbf{1 . 3}$ & $\mathbf{2 . 1}$ & $\mathbf{2 . 2}$ & $\mathbf{2 . 3}$ \\
\hline L & K & E & D & Q & T \\
\hline A & I & P & O & R & C \\
\hline M & N & U & Y & D & R \\
\hline I & P & A & W & T & W \\
\hline X & H & N & B & W & O \\
\hline K & A & L & T & Z & S \\
\hline U & X & M & Q & O & F \\
\hline G & G & H & V & S & Q \\
\hline N & E & K & F & B & B \\
\hline O & L & J & Z & C & D \\
\hline H & J & I & C & V & V \\
\hline E & M & X & S & Y & Z \\
\hline J & U & G & R & F & Y \\
\hline
\end{tabular}

\begin{tabular}{|c|c|c|c|c|c|}
\hline Set & Set & Set & Set & Set & Set \\
$\mathbf{1 . 1}$ & $\mathbf{1 . 2}$ & $\mathbf{1 . 3}$ & $\mathbf{2 . 1}$ & $\mathbf{2 . 2}$ & $\mathbf{2 . 3}$ \\
\hline 8 & 4 & 7 & 8 & 1 & 0 \\
\hline 2 & 6 & 3 & 1 & 0 & 7 \\
\hline 9 & 1 & 4 & 0 & 6 & 6 \\
\hline 1 & 2 & 9 & 7 & 5 & 8 \\
\hline 7 & 0 & 5 & 4 & 9 & 9 \\
\hline 4 & 5 & 7 & 6 & 1 & 2 \\
\hline 0 & 7 & 6 & 2 & 8 & 3 \\
\hline 6 & 8 & 1 & 5 & 2 & 8 \\
\hline 3 & 2 & 8 & 0 & 7 & 1 \\
\hline 7 & 8 & 0 & 9 & 3 & 6 \\
\hline 5 & 4 & 2 & 3 & 5 & 4 \\
\hline 1 & 3 & 9 & 5 & 4 & 2 \\
\hline 3 & 9 & 3 & 4 & 6 & 5 \\
\hline
\end{tabular}

Figure 3. Set orders by Set (1 or 2$)$ and Sub-set $(0.1,0.2,0.3)$ for letters and digits. 


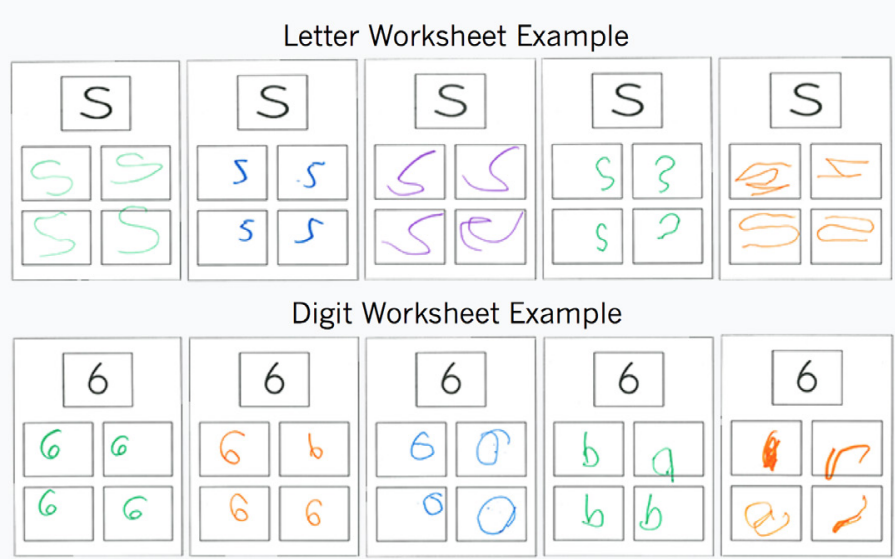

Figure 4. Examples of letter- and digit-writing worksheets.

font centered within a $3.25 \times 2.75$ inch box located centrally in the upper third of $8 \times 10$ inch workbook pages. Four blank $3.25 \times 2.75$ inch boxes were located below the typed letter, and children were asked to copy the typed exemplar above into them (see Figure 4). Each child practiced 13 symbols at each training session two times per week, so they did not become overwhelmed in a single session. There were six sets of worksheets each for the letter-writing and the digit-writing groups, two sets (set1, set2) of 13, rearranged three different ways $(0.1,0.2,0.3)$ so that each child was given a different order of symbols each week (see Figure 3). Participants completed 12 worksheets (two per week) throughout the sixweek training, cycling through the six sets twice throughout the training period. The worksheets were given in the same order to each subject, and the two sets were given in reverse order for the second three weeks (weeks 4-6) to avoid order effects (week 1: 1.1, 2.1; week 2: 1.2, 2.2; week 3: 1.3, 2.3; week 4: $2.1,1.1$; week $5: 2.2,1.2$; week $6: 2.3,1.3$ ). The experimenter pointed to the top of each worksheet and then pointed to the boxes below saying, "Make this letter (or number) in the boxes below." The experimenter never named the letters or digits.

Post-test. During the eighth week, all participants completed both the Standardized Testing Session and the Symbol Knowledge Testing Sessions again. The only difference between pre-testing and post-testing procedures is the categorization task for the letter-writing group. In the pre-test, both letter- and digit-writing groups sorted nine Zaner-Bloser typed letters and 17 handwritten letters. In the post-test, the letter-writing group sorted nine Zaner-Bloser typed letters, nine handwritten letters, and eight of their own handwritten letters produced in their last training session.

\section{ANALYSIS}

All analyses were performed with SPSS statistics $₫$ software.

\section{Description of Sample}

Sample characteristics were determined by examining the distribution of the standardized assessments and letter knowledge test scores from the pre-test session. All test scores are reported as percent correct. Scores from the Letter

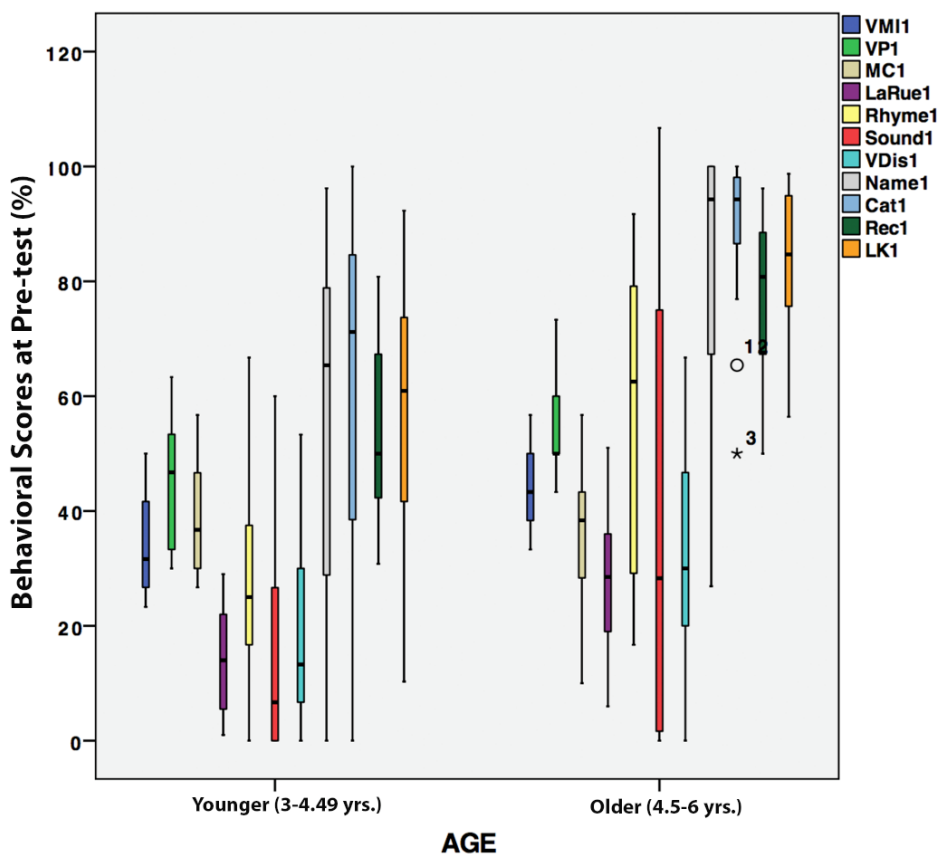

Figure 5. Box plots showing the distribution of the data in behavioral scores at pre-test by age.

Mean LETTER KNOWLEDGE by AGE and CONDITION

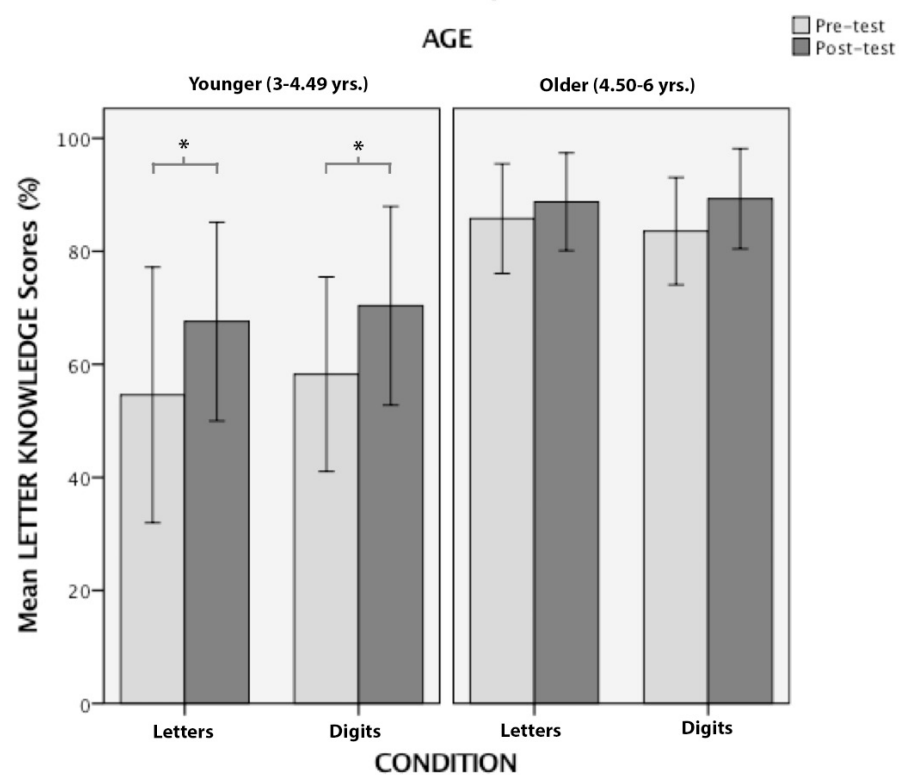

Figure 6. Bar graph showing mean letter knowledge by age and condition. Error bars indicate 95\% confidence interval, and * indicates significant effect.

Naming, Letter Recognition, and Letter Categorization tasks were averaged to create the letter knowledge composite score.

We also screened for outliers by examining the spread of each of the pre-test scores about the median. Outliers were defined as data points that lie outside of the extreme bounds of the scores sampled, which was quantified as 1.5 times the inter-quartile range. This method was chosen because no assumptions are made about the distribution of the data. 
Box plots were constructed for visualization purposes (see Figure 5).

\section{Analysis of Training Effects on Letter Knowledge}

We performed a 2x2x2 repeated measures ANOVA with letter knowledge as the dependent variable. Factors included TIME (pre, post), AGE (younger: 3-4.49 yrs., older: 4.5-6 yrs.), and CONDITION (letter-writing, digit-writing). Post hoc paired t-tests were performed to look into interaction effects revealed by the omnibus ANOVA. This analysis excluded any outliers found in the above outlier analysis. Bar plots were constructed for visualization purposes (see Figure 6).

\section{RESULTS}

\section{Sample Characteristics}

The younger group $(M=3.98, S D=.40)$ and the older group $(M=5.16, S D=.41)$ both included 16 participants. The letter-writing group included 17 participants $(M$ $=4.66$ yrs., $S D=.76)$, and digit-writing group included 15 participants $(M=4.46$ yrs., $S D=.68)$. Pre-test data indicated that our sample was normally developing based on visual perception, motor coordination, visual-motor integration, phonological processing skills, and pre-literacy level. One outlier was identified in the older group. Because the outlier did not affect the results when the ANOVA was reanalyzed, we excluded it from the results reported below.

\section{Analysis of Training Effects on Letter Knowledge}

The omnibus ANOVA revealed a significant main effect for TIME, $F(1,27)=22.80, p<.05$. All participants displayed greater letter knowledge at post-test $(M=78 \%$, $S D=19 \%)$ than at pre-test $(M=70 \%, S D=23 \%)$. There was a significant TIME $x$ AGE interaction, $F(1,27)=5.36$, $p<.05$. The younger group showed a significant increase, $13 \%$ on average, in letter knowledge over the pre-post testing periods than the older group, $4 \%$ on average (see Figure 6). There was not a significant TIME x CONDITION interaction, $F(1,27)=.071, p=.79$. Neither group, letterwriting nor digit-writing, improved significantly more than the other. There was no significant three-way interaction, $F(2,21)=.27, p=.61$. There was also no ceiling effect in the older group because their average pre-test letter knowledge score was $84 \%$.

\section{DISCUSSION}

We found that younger children showed a significantly higher increase in letter knowledge than older children over the course of a six-week training period, suggesting that if given more training time, younger children can benefit from fine-motor practice with symbols. However, this result was independent of the type of symbol practice (letters or digits), suggesting that any motor practice during this time period increases letter recognition.

Longcamp et al. (2005) demonstrated that practice printing letters improves letter recognition abilities more than practice typing letters in preschool-aged children; however, their results indicate that printing practice is most effective for five-year-old children with no significant improvements in the three- and four year-old children (Longcamp et al., 2005). We have suggested that practice printing letters provides children with variable exemplars of each letter category and facilitates letter recognition. In writing a letter multiple times, children create for themselves varying examples of each letter category, the variability of which narrows with experience. By this hypothesis, given enough practice, younger children should show an increase in letter recognition ability more than older children (who produce less variable forms); this idea was supported by the current study.

Importantly, experience with a narrow range of variable exemplars is different than experience with a wide range of variable exemplars, indicating that the range of variability experienced may have different effects on subsequent recognition. Narrow-range experience improves recognition and retention, whereas wide-range experience improves generalization (Twomey \& Horst, 2011; Twomey et al., 2013). Object recognition is the identification of an object and is distinct from object naming, which is the ability to assign a label to an object; object retention is the ability to recognize a previously learned object after a delay period. Object generalization is the ability to identify an object as belonging to a particular object category despite deviations from the category stereotype and is typically thought to be a task requiring a more experienced perceptual system than object recognition and retention. For example, cups vary greatly in their size, shape, texture, weight, and color. Nonetheless, humans can easily tell, despite these variations, that the object is still a cup. Just as children recognize and retain more object categories after experience with variable exemplars of common handheld objects, children recognize and retain more written symbol categories after experience with variable exemplars of those symbol categories ( $\mathrm{Li}$ \& James, in press). Therefore, preschool children with little to no experience with letters should show a similar pattern in learning to recognize letters after experience with narrow-range and wide-range variable exemplars: narrow variability early on should aid recognition and retention, whereas widely varying exemplars should hamper recognition and retention in preschool children. However, the developmental progress of handwriting is such that widely varying exemplars are encountered first, the range of which decreases with experience, rendering the beneficial effect of handwriting more apparent in younger children.

Pre-literate children with immature fine motor skills produce highly variable exemplars, beyond the bounds of the narrow variability required for the acquisition and retention of categories (Twomey \& Horst, 2011; Twomey et al., 2013). However, preschoolers' productions of diagnostic features are within the range of narrow variability (Beery et al., 2010). Symbols within an orthography's writing 
system make use of recurrent features, such as curve, rotation, reversal, and perspective (E. Gibson, J. Gibson, Pick, \& Osser, 1962). Thus, we suspect that symbol recognition is facilitated by perceptual gains for recurrent features because symbol recognition should be hampered if children are focusing on the entire symbol-form due to the extreme variability but should be facilitated if children are focusing on diagnostic features. Perhaps, the variability inherent in the experience of copying symbol forms, such as letters or digits, improves the recognition of forms within that symbol category or whether the experience improves written form recognition more broadly within that writing system.

Our finding that both letter-writing and digit-writing groups improved with training suggests that the benefit for letter recognition is not specific to the form being practiced. We consider three, not mutually exclusive, reasons for this result. First, any fine-motor practice may help children learn letters. Second, any training at all over the six-week period would facilitate letter learning. Third, recurrent features that are apparent in both digits and letters facilitate category understanding of letters. Fine-motor practice has been shown to help children better recognize letters. But will any type of fine motor practice improve letter leaning? Practice handwriting a letter has been shown to increase letter recognition in children over the more gross motor function of typing, and letterwriting has been correlated with letter naming (Longcamp, et al., 2005; Molfese et al., 2006). The current study found that digit-writing can be just as beneficial to letter knowledge as letter-writing. Would other forms of fine -motor control, other than writing symbols, aid in a similar increase in letter knowledge? Further research is required to answer this question. Perhaps the increase in letter knowledge simply stems from the duration of practice. The current study found that younger children benefit more from symbol-writing over the course of six weeks. It might be that any symbol training over six weeks of practice would yield a similar result. We are currently testing if symbol-viewing will have the same effects. The children in the symbol-viewing condition are exposed to letters or digits for the same amount of time on average so that we can compare the letter-writing, letter-viewing, digitwriting, and digit-viewing conditions to examine if any symbol practice would improve letter knowledge over six weeks.

The younger children might have outperformed the older children because the older children had no room for improvement from pre-test to post-test. However, the mean scores of the older children indicate an average of 84 percent on the pre-test, suggesting that both the younger and older groups had the capability for improvement in letter knowledge from pre-test to post-test. Another limitation of these findings is the lack of control over effort. That is, the worksheets might not have been exciting enough to maintain attention to the task. For example, children chose to scribble when they determined they did not know how to reproduce a letter. This would happen in the younger children more than the older children. If there was an effect of effort, the younger children would show less improvement than the older children; but because we found the opposite effect, effort most likely did not affect these results. It was also difficult to control for outside practice of letters. Preschool-aged old children are constantly surrounded by letters and digits both at school and at home. We suggest that this outside practice would be distributed among all participants and would therefore not show an overall effect on the data. It should also be noted that the data analyzed here is a subset of a larger study focusing on how fine-motor practice affects letter knowledge, within which correlations among our standardized tests and results from the complete design will be assessed.

\section{CONCLUSION AND IMPLICATIONS}

This study suggests that having pre-literate children practice copying symbols before the age of four and a half will significantly improve letter knowledge. Perhaps this increased letter knowledge will aid in learning phonological sounds and future reading ability.

\section{AUTHOR INFORMATION}

All correspondence should be sent to dzemlock@indiana.edu.

\section{ACKNOWLEDGMENTS}

I would like to thank Dr. Karin James and Sophia Vinci-Booher for their guidance and support on this research project. This work was supported by NIH (NICHD) T32 Grant \#HD 007475 awarded to SVB and KHJ.

\section{REFERENCES}

Bader, L. A. (1983). Bader Reading and Language

Inventory. Macmillan.

Beery, K. E., Buktenica, N. A., \& Beery, N. A. (2010). The Beery-Buktenica developmental test of visual-motor integration: Administration, Scoring, and Teaching Manual (Eds.). Minneapolis, MN: NSC Pearson.

Dodd, B. (2008). The Preschool and Primary Inventory of Phonological Awareness. London, UK: Psychological.

Gibson, E. P., Gibson, J. J., Pick, A. D., \& Osser, H. (1962). A developmental study of the discrimination of letterlike forms. Journal of Comparative and Physiological Psychology, 55(6), 897-906.

Goldstone, R. L. (1994). The role of similarity in categorization: Providing a groundwork. Cognition, 52(2), 125-157.

Goldstone, R. L., Medin, D. L., \& Gentner, D. (1991). Relational similarity and the nonindependence of features in similarity judgments. Cognitive Psychology, 23(2), 222-262. 
Hernandez, D. (2012). Double Jeopardy: How Third Grade Reading Skills and Poverty Influence Graduation Rates. Retrived from The Annie C. Casey Foundation: http:// gradelevelreading.net/wp-content/uploads/2012/01/ Double-Jeopardy-Report-030812-for-web1.pdf

Horst, J. S., Twomey, K. E., \& Ranson, S. L. (2013). Encountering multiple exemplars during fast mapping facilitates word learning. In Proceedings of the $35^{\text {th }}$ Annual Conference of the Cognitive Science Society (pp. 2566-2571). Austin, TX: Cognitive Science Society.

James, K. H. (2010). Sensorimotor experience leads to changes in visual processing in the developing brain. Developmental Science, 13(2), 279-288.

James, K. H., \& Engelhardt, L. (2012). The effects of handwriting experience on functional brain development in pre-literate children. Trends in Neuroscience and Education, 1(1), 32-42.

James, K. H., \& Gauthier, I. (2006). Letter processing automatically recruits a sensory-motor brain network. Neuropsychologia, 44(14), 2937-2949.

LaRue, Charles. "LaRue Literacy Tests." Retrieved from OER Commons - Institute for the Study of Knowledge Management in Education: https://www.oercommons. org/authoring/5781-larue-literacy-tests/view

Li, J. \& James, K.H. (in press). Symbol learning is facilitated by the visual variability produced by handwriting. Journal of Experimental Psychology: General.

Longcamp, M., Anton, J. L., Roth, M., \& Velay, J. L. (2003). Visual presentation of single letters activates a premotor area involved in writing. Neuroimage, 19(4), 1492-1500.

Longcamp, M., Zerbato-Poudou, M., \& Velay, J. (2005). The influence of writing practice on letter recognition in preschool children: A comparison between handwriting and typing. Acta Psychologica, 119(1), 67-79.

Molfese, V. J., Beswick, J., Molnar, A., \& Jacobi-Vessels, J. (2006). Alphabetic skills in preschool: A preliminary study of letter naming and letter writing. Developmental Neuropsychology, 29(1), 5-19.

National Assessment of Educational Progress (2013). Report on Reading Progress.

Pelatti, C. Y., Piasta, S. B., Justice, L. M., \& O'Connell, A. (2014). Language-and literacy- learning opportunities in early childhood classrooms: Children's typical experiences and within-classroom variability. Early Childhood Research Quarterly, 29(4), 445-456.

Scanlon, D. M., \& Vellutino, F. R. (1996). Prerequisite skills, early instruction, and success in first grade reading: Selected results from a longitudinal study. Mental Retardation and Development Disabilities, 2, 54-63.
Snow, C. E., Burns, M. S., \& Griffin, P. (1998). Preventing Reading Difficulties in Young Children Committee on the Prevention of Reading Difficulties in Young Children. Washington, DC: National Research Council.

Twomey, K. E., \& Horst, J. S. (2011). All things considered: Dynamic field theory captures effect of categories on children's word learning. Computational Models of Cognitive Development. Symposium conducted at the meeting of the Society of Articficial Intelligence and the Simulation of Behaviour, UK: University of York.

Twomey, K., Ranson, S., \& Horst, J. (2013). That's more like it: Multiple exemplars facilitate word learning. Infant and Child Development, 23(2), 105-122. 